\title{
The Effects of a Short-term Aerobic Training on Biochemical Cardiovascular Risk Factors and IL-6 in Obese Females
}

\author{
Zohreh Afsharmand ${ }^{1}$, Mojtaba Eizadi ${ }^{*}$, Maryam Farbod ${ }^{3}$, Nader Shakeri ${ }^{4}$ \\ 'Department of Physical Education and Sport Sciences, Islamshahr Branch, Islamic Azad University, Tehran, Iran \\ ${ }^{2}$ Department of Exercise Physiology, Saveh Branch, Islamic Azad University, Saveh, Iran \\ ${ }^{3}$ Department of Physical Education and Sport Sciences, Parand Branch, Islamic Azad University, Tehran, Iran \\ ${ }^{4}$ Department of Exercise Physiology, Science and Research Branch, Islamic Azad University, Tehran, Iran
}

\author{
*Correspondence to \\ Eizadi Mojtaba, \\ Department of Exercise Physiology, \\ Saveh Branch, Islamic Azad \\ University, Saveh, Iran. \\ Tel: 09193551960 ; \\ Fax: 086-42433007; \\ Email: \\ izadimojtaba2006@yahoo.com
}

Received June 10, 2017 Accepted October 15, 2017 Published online December 31, 2017

\begin{abstract}
Introduction: Obesity participates in the progression of systemic inflammation and cardiovascular disorders. We aimed to assess the impacts of aerobic training on serum interleukin-6 (IL-6) and risk factors for cardiovascular dysfunction in obese women.

Methods: Physically inactive, obese women matched for age (38 \pm 5 years) and body mass index (BMI) $\left(30 \leq \mathrm{BMI} \leq 36 \mathrm{~kg} / \mathrm{m}^{2}\right)$ were randomly divided into exercise (moderate aerobic training, 6 weeks, $3 \mathrm{~d} / \mathrm{wk}, \mathrm{n}=13$ ) and control (no training, $\mathrm{n}=13$ ) groups. Pre- and post-training measurements of anthropometric markers, serum IL-6 and cardiovascular risk factors were done. Within and between groups comparisons were performed by paired sample t-test and independent sample $t$ test, respectively.

Results: Aerobic training induced significant decreases in body weight, BMI, body fat (\%) and abdominal obesity as anthropometric markers $(P<0.05)$. Compared to pre-training condition, serum IL-6, triglyceride (TG), total cholesterol (TC) and Low-density lipoprotein cholesterol (LDL-C) showed no alterations by aerobic training in the exercise group $(P>0.05)$. However, participants in the aerobic training group revealed a significant increase in high-density lipoprotein cholesterol (HDL-C) and a decrease in TG/HDL and LDL/HDL $(P<0.05)$.

Conclusion: Short-time aerobic training does not appear to contribute to circulating serum levels of IL-6 in obese females; however, it affects lipid profile and may be considered as a beneficial non-pharmaceutical intervention against cardiovascular diseases in obese women. Keywords: Aerobic training, Cardiovascular risk factor, Inflammation, Obesity
\end{abstract}

Please cite this article as follows: Afsharmand Z, Eizadi M, Farbod M, Shakeri N. The effects of a short-term aerobic training on biochemical cardiovascular risk factors and IL-6 in obese females. Int J Basic Sci Med. 2017;2(4):194199. doi:10.15171/ ijbms.2017.36.

\section{Introduction}

Although adipose tissue has significant benefits, especially in hunger, obesity is associated with significant health problems. Increased adipose tissue results in physiological and morphological changes, including exudation of macrophages and release of numerous proinflammatory cytokines, some of which directly or indirectly influence insulin sensitivity by regulating insulin signaling and molecules involved in fat and glucose metabolism. ${ }^{1}$ Increased adipose tissue in old age or increased secretion of anti-inflammatory cytokines from mononuclear blood cells leads to reduced muscle mass. ${ }^{2}$

Cytokines are peptides or proteins that are secreted into the bloodstream by immune system. ${ }^{2}$ Interleukin-6 (IL-6) is one of the cytokines secreted by adipocytes and some other tissues which has both pro- and anti-inflammatory roles. ${ }^{3}$ It is known that systemic concentrations of IL-6 increase in the obesity and type 2 diabetes. It has also been found that IL- 6 concentration in tissue and serum is associated with cell metabolism. ${ }^{4}$ Some studies have indicated that elevation of IL- 6 predicts mortality and morbidity rates in elderly. ${ }^{5}$ The active form of IL-6 is a homodimer, each subunit of which is a globular domain with four alpha helixes. Mononuclear phagocytes, vascular endothelial cells, fibroblasts, and other cells produce and secrete IL- 6 in response

(c) 2017 The Author(s); Published by Zabol University of Medical Sciences. This is an open-access article distributed under the terms of the Creative Commons Attribution License (http://creativecommons.org/licenses/by/4.0), which permits unrestricted use, distribution, and reproduction in any medium, provided the original work is properly cited. 
to microbes and other cytokines. ${ }^{6}$ It has been found that high plasma IL- 6 concentrations are accompanied by a reduction in muscular mass and function. ${ }^{7}$

Some recent studies stated that circulating levels of inflammatory cytokines such as IL-6 varies depending on the amount of adipose tissue. ${ }^{8}$ It is believed that weight loss, especially in obese people, is accompanied by improved inflammatory and metabolic profiles. In this regard, some studies have reported that inflammatory profile improves following short- and long-term training programs. In a recent study, 12 aerobic/resistance training resulted in a significant decrease in serum tumor necrosis factor-alpha (TNF- $\alpha$ ) and IL-6 in middle-aged obese men. ${ }^{9}$ In another report, 1-year weight loss program was accompanied by improved cardiovascular risk factors. ${ }^{10}$ Nevertheless, 6-month aerobic training program did not result in any changes in proinflammatory cytokines such as IL-6, C-reactive protein (CRP), and TNF- $\alpha$ in obese or overweight postmenopausal women. ${ }^{11}$ In another study, a 12-week aerobic training program did not cause any changes in the levels of cardiovascular risk factors such as cholesterol, triglyceride, and HDL in obese women with metabolic syndrome. ${ }^{12}$ However, inflammatory mediators such as IL-6 improved following 16-weeks aerobic training and respiratory muscle training program. ${ }^{13,14}$ A limited number of studies have focused on the importance of aerobic exercise in modulating levels of IL-6 and cardiovascular markers in obese women. Based on this contradiction, this study attempted to assess the impacts of a 6-week aerobic training on serum levels of IL- 6 as an inflammatory cytokine and cardiovascular risk factors in sedentary obese women.

\section{Methods}

\section{Study Subjects}

Participants of this semi-experimental study included 26 sedentary obese pre-menopausal women matched for age (38 \pm 5 years) and body mass index (BMI) $\left(32 \pm 3 \mathrm{~kg} / \mathrm{m}^{2}\right)$ that participated by convenience sampling. The subjects were randomly allocated into aerobic training $(n=13)$ or control $(n=13)$ groups.

\section{Inclusion and Exclusion Criteria}

Inclusion criteria for subjects were: being healthy, being middle-aged, and having obesity $(30 \leq \mathrm{BMI} \leq 36)$. Participants were non-alcoholics, non-athletes (VO2max $\leq 27 \mathrm{~mL} / \mathrm{kg} / \mathrm{min}$ ) and non-smokers. Participants did not participate in any regular physical activity or follow any specific diet within the past 6 months. The exclusion criteria included a history of diabetes, acute or chronic respiratory infections, cardiopulmonary and neuromuscular disease.

\section{Anthropometry}

Anthropometric indexes were measured for each subject. Obesity was determined by BMI (kg) divided by height squared $\left(\mathrm{m}^{2}\right)$. The height and weight were measured by the same person for each subject. The height and weight measurements were carried out with the least amount of clothes on with no shoes. Abdominal and hip circumference and the ratio between them (AHO) were documented in standing position. Body composition monitor (OMRON, Finland) used to measure body fat (\%).

\section{Exercise Program}

Aerobic training was performed for 6 weeks, 3 sessions per week. The protocol included warming up (10 minutes), and then 30 to 45 minutes of the main exercise at 60 to $70 \%$ of maximum heart rate followed by a coolingdown period. ${ }^{15}$ The training activity included walking or running on a treadmill without slope. Moreover, subjects in the exercise group were advised to do regular physical activities such as brisk walking and so on. The exercise intensity was up to $60 \%$ of maximum heart rate in the first week and then was gradually increased during the study period. For each subject, exercise intensity decreased by $5 \%$ during menstrual cycles. ${ }^{16}$ Control subjects were asked to continue their daily routine activities. Participants were also requested to follow their usual diet.

\section{Biochemical Analysis and Assay}

Blood samples were taken from each subject considering a 12-hour overnight fasting period. They were requested to avoid vigorous physical activity 48 hours before sampling. Post-training blood samples were collected 48 hours after the last exercise session. Serums were separated and stored at $-80^{\circ} \mathrm{C}$ until use. Serums were used to measure IL- 6 and biochemical cardiovascular risk factors. ELISA method was used for determining serum IL-6 (BiovendorLaboratorial kit made by Biovendor Company, Czech). Coefficients of variation (CV) of the kit were 3.4\% (intraassay) and 5.2\% (inter-assay), respectively. TG, TC, HDL and LDL cholesterol were measured using the colorimetric enzymatic method (Pars Azmoon, Tehran, Iran).

\section{Data Analysis}

Data was analyzed using SPSS statistical software package version 16.0 (SPSS Inc., IL, USA). Normal distribution was checked by Kolmogorov-Smirnov test. Comparisons of each variable between the 2 groups were done using independent sample $t$ test. Comparisons between pre and post-training measures were done by paired sample $t$ test. A $P$ value of less than 0.05 was considered statistically significant.

\section{Results}

Table 1 shows the anthropometric features at the pre and post-training time points. All values have been reported as mean and standard deviation (SD). Based on the results of independent sample $t$ test, no statistically significant differences were found between the 2 studied 
Table 1. Comparison of Descriptive Anthropometric Features Before and After the Aerobic Intervention in Exercise and Control Groups

\begin{tabular}{|c|c|c|c|c|c|c|}
\hline \multirow{2}{*}{ Variables } & \multicolumn{3}{|c|}{ Exercise Group } & \multicolumn{3}{|c|}{ Control group } \\
\hline & Pre-training & Post-training & $P$ & Pre-training & Post-training & $P$ \\
\hline Age $(y)$ & $38.4 \pm 5.65$ & - & - & $37.3 \pm 2.39$ & - & - \\
\hline Height (cm) & $162 \pm 4.86$ & - & - & $161 \pm 4.66$ & - & . \\
\hline Weight (kg) & $83.4 \pm 8.45$ & $82 \pm 8.56$ & 0.038 & $83.7 \pm 4.81$ & $83.6 \pm 4.71$ & 0.263 \\
\hline $\mathrm{AC}(\mathrm{cm})$ & $107 \pm 7.4$ & $106 \pm 8$ & 0.031 & $108 \pm 8$ & $108 \pm 11$ & 0.421 \\
\hline $\mathrm{HC}(\mathrm{cm})$ & $104 \pm 11$ & $102 \pm 13$ & 0.009 & $105 \pm 10$ & $105 \pm 9$ & 0.356 \\
\hline BMI (kg/m2) & $31.8 \pm 2.76$ & $31.2 \pm 2.80$ & 0.024 & $32.3 \pm 1.58$ & $32.25 \pm 1.57$ & 0.723 \\
\hline $\mathrm{BF}(\%)$ & $44.7 \pm 4.55$ & $44 \pm 4.33$ & 0.016 & $44.1 \pm 2.95$ & $43.5 \pm 2.62$ & 0.543 \\
\hline
\end{tabular}

Abbreviations: AC, abdominal circumference; HC, hip circumference; $\mathrm{BMI}$, body mass index, BF, body fat percentage.

groups at baseline $(P>0.05)$. On the other hand, aerobic intervention induced a significant decrease in body weight, BMI, abdominal obesity and body fat (\%) in exercise groups but these variables remained unchanged in control subjects $(P<0.05$; Table 1$)$.

At baseline (pre-training), serum IL-6 levels were not different between the two groups $(P=0.423)$. Despite the reduction in anthropometric indexes, no significant alteration was noticed in serum IL-6 level after aerobic intervention compared to pre-training levels in the aerobic exercise group $(P=0.092)$. There were no differences in the cardiovascular risk factors between the 2 groups at baseline $(P>0.05)$. No differences in TC, TG and LDL cholesterol were found after aerobic training compared to their respective baseline values in the exercise groups $(P>0.05)$. In contrast, HDL cholesterol was significantly increased by aerobic training in the exercise group $(P=0.022)$ but not in control subjects $(P=0.723)$. In addition, compared to pre-training, TG/HDL $(P=0.011)$ and LDL/HDL $(P=0.009)$ decreased significantly in exercise subjects, but not in the control subjects $(P>0.05)$ (Table 2 and Figure 1).

\section{Discussion}

We found that serum levels of IL-6, which is an inflammatory cytokine, did not change following 6 weeks of aerobic training in obese women. Although the training intervention was associated with a decline in serum IL-6, this reduction was not statistically significant; however, these changes were remarkable from a clinical perspective. Moreover, this program was not accompanied by any changes in the levels of TG, TC, and LDL as cardiovascular risk factors, although serum levels of HDL significantly declined. Most studies support higher levels of inflammatory cytokines in the presence of obesity. ${ }^{17}$

The role of inflammatory markers in the etiology of diabetes has been proposed as well as the potential effects of IL-6 and CRP in determining markers of metabolic syndrome and type $2 .^{1}$ IL- 6 as an active inflammatory cytokine is produced by many cells such as leukocytes, adipocytes, and endothelial cells. ${ }^{18}$ Researchers believe that IL-6 is related to hyperglycemia, insulin resistance, and type 2 diabetes as a critical physiological marker sensitive to systemic inflammation. ${ }^{1}$ This inflammatory marker has also been introduced as a predictor of cardiovascular diseases. ${ }^{19}$ Injection of IL- 6 to healthy people or patients with type 2 diabetes increases lipolysis without inducing the elevation of triglyceride or changes in catecholamines, glucagon, and insulin. ${ }^{20}$ Suppression of IL-6 under clinical conditions leads to increased cholesterol and higher glucose levels. ${ }^{21}$ Moreover, several studies mentioned that IL-6 can be involved in the oxidation of free fatty acids in intracellular spaces and in the whole body. ${ }^{20}$ Similar to inflammatory profile, higher levels of blood lipids have been reported in obese people. ${ }^{22}$

Table 2. Comparison of Serum IL-6 and Cardiovascular Risk factors Before and After the Aerobic Intervention in Exercise and Control Groups

\begin{tabular}{|c|c|c|c|c|c|c|}
\hline \multirow{2}{*}{ Variables } & \multicolumn{3}{|c|}{ Exercise Group } & \multicolumn{3}{|c|}{ Control group } \\
\hline & Pre-training & Post-training & $\boldsymbol{P}$ & Pre-training & Post-training & $\boldsymbol{P}$ \\
\hline Interleukin-6 (pg/mL) & $6 \pm 1.49$ & $5.4 \pm 1.46$ & 0.092 & $5.9 \pm 1.40$ & $5.7 \pm 1.03$ & 0.412 \\
\hline $\mathrm{TC}(\mathrm{mg} / \mathrm{dL})$ & $168 \pm 33$ & $157 \pm 44$ & 0.321 & $150 \pm 28$ & $159 \pm 33$ & 0.524 \\
\hline $\mathrm{TG}(\mathrm{mg} / \mathrm{dL})$ & $123 \pm 85$ & $119 \pm 44$ & 0.073 & $127 \pm 73$ & $130 \pm 61$ & 0.362 \\
\hline LDL (mg/dL) & $112 \pm 26$ & $108 \pm 29$ & 0.112 & $118 \pm 24$ & $123 \pm 33$ & 0.264 \\
\hline HDL (mg/dL) & $41 \pm 9.6$ & $44.3 \pm 6.8$ & 0.022 & $40 \pm 6.7$ & $41 \pm 5.9$ & 0.723 \\
\hline TG/HDL & $3 \pm 1.21$ & $2.67 \pm 0.93$ & 0.011 & $3.18 \pm 1.12$ & $3.17 \pm 1.23$ & 0.232 \\
\hline LDL/HDL & $2.73 \pm 0.51$ & $2.44 \pm 0.59$ & 0.009 & $2.95 \pm 0.73$ & $3 \pm 1.12$ & 0.412 \\
\hline
\end{tabular}

Abbreviations: TC, total cholesterol; TG, triglyceride; LDL, low density lipoprotein; HDL, high density lipoprotein. 
A

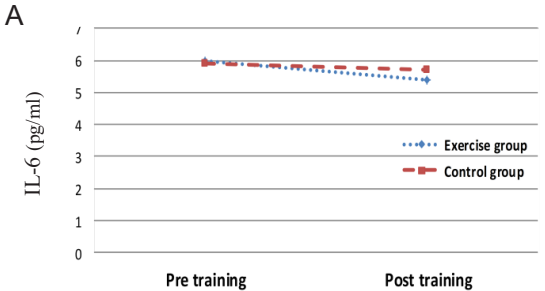

C

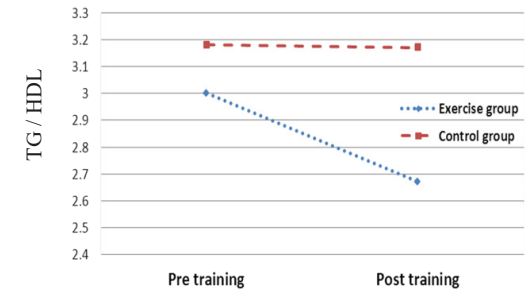

B

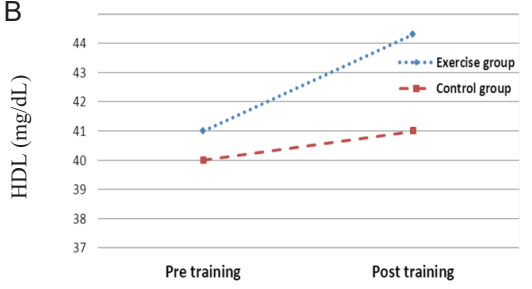

D

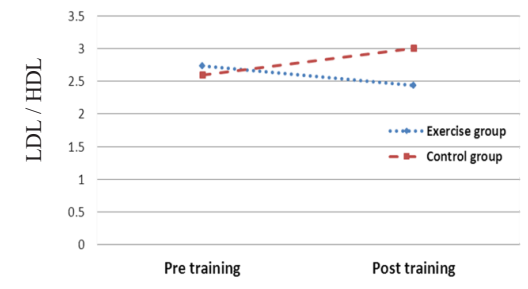

Figure 1. Profiles of the Effects of 6 Weeks of Aerobic Training on Serum IL-6 (A), HDL (B), TG/HDL (C) and LDL/HDL (D) in Studied Obese Females

The researchers believe that weight loss or reduction of body fat is associated with the improvement of cardiovascular risk factors in obese populations. ${ }^{22}$ For example, in one study, a weight loss of 11 kilograms led to improved levels of LDL and TC as cardiovascular risk factors. ${ }^{10}$ Improved lipid profile markers following aerobic training have also been reported by some other studies. ${ }^{23}$ Nevertheless, there are studies reporting that cardiovascular risk factors did not change following short or long-term training programs in obese populations. ${ }^{24}$ The response of inflammatory cytokines to exercise or diet interventions in healthy or sick populations is often contradictory. Although the main mechanisms responsible for this paradox have not been completely known yet, this contradiction can be somehow attributed to the training program in terms of duration, frequency, and volume of exercise, measurement tools, and the study population. In the present study, the short-term training program may have been one of the factors responsible for the lack of improvement in IL-6 or cardiovascular risk factors.

Despite the significant weight loss observed in response to the 6-week aerobic training in this study, some studies have considered a minimum of $10 \%$ reduction in body weight in response to training programs as a sufficient condition for improvements in inflammatory cytokines or cardiovascular risk factors in obese populations. ${ }^{25}$ However, in one study, a short-term (4 weeks) training led to the reduction of cardiovascular risk factors along with the increase in HDL. ${ }^{26}$ Moreover, some studies have not reported a reduction in body weight or body fat percentage. In one of these studies, despite the lack of changes in BMI or body fat percentage, the researchers reported a significant reduction in inflammatory cytokines in the elderly. ${ }^{27}$

Older people, regardless of the type of disease, suffer systemic inflammation, and numerous studies have indicated a link between physical activity and inflammatory mediators in this population. ${ }^{28}$ Therefore, it is believed that higher levels of inflammation in old people is an appropriate explanation for the greater response of the inflammatory profile in them in response to exercise training, even in the absence of the change in weight or body fat. Accordingly, it is assumed that, apart from variations in weight and body fat, the gradual increase of age is effective in the improvement of the inflammatory profile in response to exercise trainings.

In other studies, a significant inverse relationship has been confirmed between the physical activity level and inflammation in young men and women. ${ }^{29}$ Some researchers also stated that the beneficial effects of exercises were not tangible without dieting. ${ }^{30}$ The researchers also believe that diet control or having a proper diet when performing exercise trainings, particularly in healthy or sick obese individuals, severely affects the inflammatory profile. ${ }^{31}$ Despite this evidence, in the study by Gueugnon et al, a 9-month program consisting of dieting and aerobic training did not significantly change serum resistin levels. ${ }^{32}$ Moreover, despite the lack of changes and without any diet control in a recent study, the 24 -week combined training (aerobic-resistance) improved the mediators of inflammation (adiponectin, leptin, resistin, and CRP) and insulin resistance in obese men, ${ }^{33}$ although serum IL-6 and IL-10 did not change.

Some of these researchers attributed the improvement in inflammatory profile to increased cardiovascular fitness resulting from training programs. ${ }^{34,35}$ In this relation, the findings of a study indicated increased levels of VO2max in response to 3 and 6 months of intensive aerobic and resistance training which led to significant improvement in cardiovascular risk factors and reduction in the inflammatory markers. ${ }^{36}$ In another study, a significant 
relationship was found between inflammatory profile, aerobic fitness, and muscular fitness of Norwegian children. ${ }^{37}$ However, some studies on healthy young and middle-aged men or women have not reported any significant correlation between the physical activity level and any inflammatory cytokines such as CRP, IL-6, and TNF- $\alpha$ receptors 1 and 2, even after adjusting for BMI. ${ }^{38}$

\section{Limitation}

We did not measure VO2max and other inflammatory cytokines in response to the training program that is considered as a limitation of our study.

\section{Conclusion}

Despite the fact that 6 weeks of aerobic training induced a significant increase in HDL in obese women, no changes were detected in other lipid indicators and IL-6. This may be due to the short duration of training. The optimal effects of this training program are important from a clinical perspective, emphasizing a significant increase in HDL and a reduction in TG/HDL and LDL/HDL.

\section{Competing Interests}

The authors declare that they have no competing interests.

\section{Ethical Approval}

Our study was approved by the Ethics Committee of the Islamic Azad University, Iran, and a written informed consent obtained from participants.

\section{Acknowledgments}

The authors would like to thank all the subjects who participated in the present study. We thank the Research Deputy of Islamic Azad University for financial support and cooperation in implementing this project.

\section{References}

1. Festa A, D’Agostino R, Jr., Howard G, Mykkanen L, Tracy RP, Haffner SM. Chronic subclinical inflammation as part of the insulin resistance syndrome: the Insulin Resistance Atherosclerosis Study (IRAS). Circulation. 2000;102(1):4247.

2. Lambert CP, Wright NR, Finck BN, Villareal DT. Exercise but not diet-induced weight loss decreases skeletal muscle inflammatory gene expression in frail obese elderly persons. J Appl Physiol (1985). 2008;105(2):473-478. doi:10.1152/japplphysiol.00006.2008

3. Gleeson M. Immune function in sport and exercise. J Appl Physiol (1985). 2007;103(2):693-699. doi:10.1152/ japplphysiol.00008.2007

4. Gustafson B, Smith U. Cytokines promote Wnt signaling and inflammation and impair the normal differentiation and lipid accumulation in 3T3-L1 preadipocytes. J Biol Chem. 2006;281(14):9507-9516. doi:10.1074/jbc. M512077200

5. Reuben DB, Cheh AI, Harris TB, et al. Peripheral blood markers of inflammation predict mortality and functional decline in high-functioning community-dwelling older persons. J Am Geriatr Soc. 2002;50(4):638-644.

6. Ferrucci L, Harris TB, Guralnik JM, et al. Serum IL-6 level and the development of disability in older persons. J Am Geriatr Soc. 1999;47(6):639-646.

7. Visser M, Pahor M, Taaffe DR, et al. Relationship of interleukin-6 and tumor necrosis factor-alpha with muscle mass and muscle strength in elderly men and women: the Health ABC Study. J Gerontol A Biol Sci Med Sci. 2002;57(5):M326-332.

8. Moschen AR, Molnar C, Geiger S, et al. Anti-inflammatory effects of excessive weight loss: potent suppression of adipose interleukin 6 and tumour necrosis factor alpha expression. Gut. 2010;59(9):1259-1264. doi:10.1136/ gut.2010.214577

9. Donges CE, Duffield R, Guelfi KJ, Smith GC, Adams DR, Edge JA. Comparative effects of single-mode vs. duration-matched concurrent exercise training on body composition, low-grade inflammation, and glucose regulation in sedentary, overweight, middle-aged men. Appl Physiol Nutr Metab. 2013;38(7):779-788. doi:10.1139/ apnm-2012-0443

10. Foster GD, Wyatt HR, Hill JO, et al. Weight and metabolic outcomes after 2 years on a low-carbohydrate versus low-fat diet: a randomized trial. Ann Intern Med. 2010;153(3):147157. doi:10.7326/0003-4819-153-3-201008030-00005

11. Lebon J, Riesco E, Tessier D, Dionne IJ. Additive effects of isoflavones and exercise training on inflammatory cytokines and body composition in overweight and obese postmenopausal women: a randomized controlled trial. Menopause. 2014;21(8):869-875. doi:10.1097/ gme.0000000000000177

12. Colombo CM, de Macedo RM, Fernandes-Silva MM, et al. Short-term effects of moderate intensity physical activity in patients with metabolic syndrome. Einstein (Sao Paulo). 2013;11(3):324-330.

13. Kadoglou NP, Perrea D, Iliadis F, Angelopoulou N, Liapis $\mathrm{C}$, Alevizos M. Exercise reduces resistin and inflammatory cytokines in patients with type 2 diabetes. Diabetes Care. 2007;30(3):719-721. doi:10.2337/dc06-1149

14. Mills DE, Johnson MA, McPhilimey MJ, et al. The effects of inspiratory muscle training on plasma interleukin-6 concentration during cycling exercise and a volitional mimic of the exercise hyperpnea. J Appl Physiol (1985). 2013;115(8):1163-1172. doi:10.1152/ japplphysiol.00272.2013

15. Eizadi M, Sokhanguei Y, Eghdami A, Banaeifar A. Effect of aerobic exercise on Pancreas Beta-cells function in adult obese males. J Birjand Univ Med Sci. 2014;21(2):203-210.

16. Mohammadi B, Azamian Jazi A, Faramarzi M, Fathollahi Shourabeh F. The Effect of Aerobic Exercise Training and Detraining on Some of the Menstrual Disorders in Nonathlete Students in Lorestan Universities. Horizon of Medical Sciences. 2012;18(2):5-12.

17. Habib SA, Saad EA, Elsharkawy AA, Attia ZR. Proinflammatory adipocytokines, oxidative stress, insulin, $\mathrm{Zn}$ and $\mathrm{Cu}$ : Interrelations with obesity in Egyptian nondiabetic obese children and adolescents. Adv Med Sci. 2015;60(2):179-185. doi:10.1016/j.advms.2015.02.002

18. Pradhan AD, Manson JE, Rifai N, Buring JE, Ridker PM. C-reactive protein, interleukin 6 , and risk of developing type 2 diabetes mellitus. Jama. 2001;286(3):327-334. 
19. Ridker PM, Rifai N, Stampfer MJ, Hennekens CH. Plasma concentration of interleukin-6 and the risk of future myocardial infarction among apparently healthy men. Circulation. 2000;101(15):1767-1772.

20. Petersen EW, Carey AL, Sacchetti M, et al. Acute IL-6 treatment increases fatty acid turnover in elderly humans in vivo and in tissue culture in vitro. Am J Physiol Endocrinol Metab. 2005;288(1):E155-162. doi:10.1152/ ajpendo.00257.2004

21. Nishimoto N, Yoshizaki K, Miyasaka N, et al. Treatment of rheumatoid arthritis with humanized anti-interleukin-6 receptor antibody: a multicenter, double-blind, placebocontrolled trial. Arthritis Rheum. 2004;50(6):1761-1769. doi:10.1002/art.20303

22. Laddu D, Dow C, Hingle M, Thomson C, Going S. A review of evidence-based strategies to treat obesity in adults. Nutr Clin Pract. 2011;26(5):512-525. doi:10.1177/0884533611418335

23. Slentz CA, Houmard JA, Johnson JL, et al. Inactivity, exercise training and detraining, and plasma lipoproteins. STRRIDE: a randomized, controlled study of exercise intensity and amount. J Appl Physiol (1985). 2007;103(2):432-442. doi:10.1152/japplphysiol.01314.2006

24. Rock CL, Flatt SW, Sherwood NE, Karanja N, Pakiz B, Thomson CA. Effect of a free prepared meal and incentivized weight loss program on weight loss and weight loss maintenance in obese and overweight women: a randomized controlled trial. Jama. 2010;304(16):18031810. doi:10.1001/jama.2010.1503

25. Dow CA, Thomson CA, Flatt SW, Sherwood NE, Pakiz B, Rock CL. Predictors of improvement in cardiometabolic risk factors with weight loss in women. J Am Heart Assoc. 2013;2(6):e000152. doi:10.1161/jaha.113.000152

26. Altena TS, Michaelson JL, Ball SD, Guilford BL, Thomas TR. Lipoprotein subfraction changes after continuous or intermittent exercise training. Med Sci Sports Exerc. 2006;38(2):367-372. doi:10.1249/01. mss.0000185088.33669.fd

27. Kohut ML, McCann DA, Russell DW, et al. Aerobic exercise, but not flexibility/resistance exercise, reduces serum IL-18, CRP, and IL-6 independent of beta-blockers, $\mathrm{BMI}$, and psychosocial factors in older adults. Brain Behav Immun. 2006;20(3):201-209. doi:10.1016/j.bbi.2005.12.002

28. Stewart LK, Flynn MG, Campbell WW, et al. Influence of exercise training and age on CD14+ cell-surface expression of toll-like receptor 2 and 4 . Brain Behav Immun. 2005;19(5):389-397. doi:10.1016/j.bbi.2005.04.003

29. Panagiotakos DB, Pitsavos C, Chrysohoou C, Kavouras S, Stefanadis C. The associations between leisure-time physical activity and inflammatory and coagulation markers related to cardiovascular disease: the ATTICA Study. Prev Med. 2005;40(4):432-437. doi:10.1016/j. ypmed.2004.07.010

30. Shahgholi Abasi R, Izadi M, Soheili S, Imanzadeh R. Serum Resistin and Insulin Resistance Responses to LongTerm Physical Exercise in the Absence of Diet Control in Middle-Aged Obese Men. J Mazandaran Univ Med Sci. 2012;21(86):126-130.

31. Bouchonville M, Armamento-Villareal R, Shah K, et al. Weight loss, exercise or both and cardiometabolic risk factors in obese older adults: results of a randomized controlled trial. Int J Obes (Lond). 2014;38(3):423-431. doi:10.1038/ijo.2013.122

32. Gueugnon C, Mougin F, Simon-Rigaud ML, Regnard J, Negre V, Dumoulin G. Effects of an in-patient treatment program based on regular exercise and a balanced diet on high molecular weight adiponectin, resistin levels, and insulin resistance in adolescents with severe obesity. Appl Physiol Nutr Metab. 2012;37(4):672-679. doi:10.1139/ h2012-045

33. Brunelli DT, Chacon-Mikahil MP, Gaspari AF, et al. Combined Training Reduces Subclinical Inflammation in Obese Middle-Age Men. Med Sci Sports Exerc. 2015;47(10):2207-2215. doi:10.1249/ mss.0000000000000658

34. Platat C, Wagner A, Klumpp T, Schweitzer B, Simon C. Relationships of physical activity with metabolic syndrome features and low-grade inflammation in adolescents. Diabetologia. 2006;49(9):2078-2085. doi:10.1007/s00125006-0320-6

35. Ruiz JR, Ortega FB, Warnberg J, Sjostrom M. Associations of low-grade inflammation with physical activity, fitness and fatness in prepubertal children; the European Youth Heart Study. Int J Obes (Lond). 2007;31(10):1545-1551. doi:10.1038/sj.ijo.0803693

36. Stavropoulos-Kalinoglou A, Metsios GS, Veldhuijzen van Zanten JJ, Nightingale P, Kitas GD, Koutedakis Y. Individualised aerobic and resistance exercise training improves cardiorespiratory fitness and reduces cardiovascular risk in patients with rheumatoid arthritis. Ann Rheum Dis. 2013;72(11):1819-1825. doi:10.1136/ annrheumdis-2012-202075

37. Steene-Johannessen J, Kolle E, Andersen LB, Anderssen SA. Adiposity, aerobic fitness, muscle fitness, and markers of inflammation in children. Med Sci Sports Exerc. 2013;45(4):714-721. doi:10.1249/MSS.0b013e318279707a

38. Pischon T, Hankinson SE, Hotamisligil GS, Rifai N, Rimm EB. Leisure-time physical activity and reduced plasma levels of obesity-related inflammatory markers. Obes Res. 2003;11(9):1055-1064. doi:10.1038/oby.2003.145 